\title{
CUATRO FUחCIONES ESTRATÉGICAS DE LA DIPLOMACIA CORPORATIVA
}

\section{Four strategic functions in corporate diplomacy}

\author{
Juan Luis Manfredi Sánchez \\ Facultad de Periodismo. Universidad de Castilla-La Mancha \\ E-mail: juan.manfredi@uclm.es
}

0 Autor

FIRMA INVITADA

La diplomacia corporativa es el desarrollo instrumental de la estrategia que ordena la relación con los poderes públicos y privados en un entorno complejo y globalizado. Las corporaciones actúan como poderes diplomáticos en la representación, la negociación, la protección y la promoción de los intereses, las cuatro funciones básicas de la diplomacia que establece la Convención de Viena de 1961. La tarea de la diplomacia corporativa es una fuente de innovación directiva y una oportunidad de desarrollo profesional.

Diplomacia corporativa; lobby; comunicación internacional; globalización.

Corporate diplomacy; lobby; international communication; globalization.

Q Key mords

Recibido: 03-04-2016. Aceptado: 15-06-2017.

Fechas

Corporate diplomacy is the instrumental development of the strategy building the relationship between public and private powers in a complex and globalized environment. International companies act as diplomatic powers in the representation, negotiation, protection and promotion of their self-interests, the four basic functions of diplomacy established by the Vienna Convention in 1961. The task of corporate diplomacy is a source of innovation management and an opportunity for professional development.

Resumen

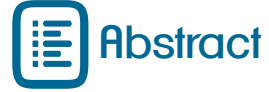




\section{Dinámica y alcance de la diplomacia corporativa}

La diplomacia consiste en la dirección y la ejecución de las relaciones internacionales por parte de países y, en un sentido más amplio, de actores con capacidad de obrar en la arena internacional. Es un instrumento que requiere competencias profesionales, habilidades, destrezas, conocimientos y actitudes para resolver con éxito las tareas asignadas en la ejecución de la actividad exterior. Es una actividad principal de la acción exterior y cuenta con normas, reglamentos, costumbres y usos que han moldeado las relaciones internacionales (Berridge, 2015). Las empresas y las organizaciones privadas han aprendido el valor de la diplomacia para la conducción de asuntos internacionales y han capturado esos aprendizajes para transformarlos en parte de las tareas asignadas a la dirección. Siguiendo a Deutsch (1978), las corporaciones están motivadas a la acción exterior por las mismas razones: preservar la seguridad y la independencia de los negocios, la protección de sus activos y la promoción de sus intereses.

La literatura académica ofrece multitud de definiciones y matices sobre en qué consiste la diplomacia corporativa, la diplomacia privada, la gestión de asuntos públicos internacionales o la gestión del capital político de la compañía. Estos y más nombres aparecen en la literatura para referirse a la misma actividad. Sondergaard (2014) establece que se trata de una función interdisciplinar que no admite un modelo general, sino que representa la suma de numerosas teorías, paradigmas y experiencias empresariales. Veamos algunas de ellas. Saner y Yiu (2005) refieren la ocupación a las compañías multinacionales que se relacionan con todo tipo de actores políticos, sociales y económicos en la ejecución de sus proyectos empresariales. Para Ordeix-Rigo y Duarte (2009), la diplomacia corporativa es sinónimo de poder y legitimidad para actuar en los mercados internacionales, en la línea de la "licencia para operar" que ha cuajado en la doctrina sobre responsabilidad empresarial. El profesor Ruël (2013) señala el análisis del entorno de negocio, la interacción con los grupos de interés y la relación con los actores diplomáticos convencionales bajo supervisión del primer ejecutivo como el compendio básico de la actividad diplomática corporativa. Lee (2004) proporciona una definición aún más amplia: "el trabajo de la red de actores públicos y privados que gestionan las relaciones comerciales mediante el empleo de canales y procesos diplomáticos" (p. 51). L’Etang (2008) vincula la actividad con las relaciones públicas en tanto que generan espacios para el diálogo con los grupos de interés, dotan de credibilidad a las compañías y manejan las herramientas propias de la negociación y la gestión de conflictos. Para la autora, son "diplomáticos organizacionales” (L’Etang, 2008, p. 239).

El conjunto ofrece una definición río, que acaudala funciones de la dirección general de las compañías que operan en un entorno internacional. Se concluye que la diplomacia corporativa es el desarrollo instrumental de la estrategia que ordena la relación con los poderes públicos y privados. Se concreta en el establecimiento de relaciones con terceros (con capacidad de acción o toma de decisiones) con el ánimo de resolver problemas propios y ajenos, la creación de marcos comunes de trabajo y la generación de confianza. Se trata, efectivamente, de trasladar un empleo que estaba reservado a las políticas de Estado a la administración de los negocios internacionales.

En la estrategia de diplomacia corporativa, confluyen tres objetivos genéricos. Persigue la legitimación de la actividad pública de las organizaciones de naturaleza privada en un entorno de creciente complejidad. La legitimidad es el desarrollo natural de la responsabilidad social, la gestión de la reputación y la imagen, así como las actividades de naturaleza política de la propia compañía (den Hond et al., 2014).

\section{La diplomacia comporativa es el desarrollo instrumental de la estrategia que ordena la relación con los poderes públicos y privados}


El segundo objetivo es la gestión de la influencia y la gestión del capital relacional de la compañía. Algunos ejemplos recurrentes son la participación en la creación de políticas públicas, la presión sobre los reguladores para que legislen en una u otra dirección, el establecimiento de relaciones comerciales internacionales o la relación con los nuevos titulares del Gobierno cuando estos cambian de forma democrática o forzosa. Saner, Yiu y Sondergaard (2008) consideran que la influencia consiste en la capacidad de crear y establecer nuevas oportunidades de negocio, trabajar con las organizaciones internacionales que marcan las reglas del comercio, prever y aminorar los conflictos con los grupos de interés y proteger la reputación a través de los medios y foros correspondientes, entre otras.

El tercer elemento es la gestión de la complejidad en la globalización (Ruël y Wolters, 2016). La tecnología, la globalización, las sociedades abiertas, el auge de la transparencia, los ciudadanos conectados, el incremento de las negociaciones multilaterales, la vuelta de la geopolítica o la incertidumbre general de los mercados ha transformado la arena internacional (Manfredi, 2014). La consolidación de los medios de comunicación internacionales, la llegada de Internet y los nuevos dispositivos móviles, la fuerza de los movimientos sociales, el impulso de los intereses corporativos globalizados o la creación de nuevas alianzas comerciales alimentan un escenario de complejidad en el que las empresas multinacionales operan. Más individuos y organizaciones participan en la toma de decisiones (Manfredi, 2011). Kesteleyn, Riordan y Ruël (2014) introducen la idea de riesgo geopolítico y riesgo de naturaleza no comercial en la actividad de la empresa. Este encuadre aparece en los proyectos docentes de las materias impartidas en universidades y escuelas de negocios anglosajonas. La agenda directiva se ha abierto a tres perspectivas convergentes: la gobernanza de las organizaciones (Huang, 2010), la gestión de la incertidumbre (Haynal, 2012) y las economías globales (Bayne y Woolcock, 2007). La inclusión de estos elementos de no mercado (Baron, 1997) conforman la estrategia tanto o más que los elementos basados únicamente en las condiciones del mercado (producto, precio, posicionamiento y promoción). Las decisiones comerciales y operativas no se sostienen si no se informa, se administra y se promueve la actividad empresarial a largo plazo.

La tarea de la diplomacia corporativa es el resultado del desarrollo de competencias y habilidades dentro de las empresas, a cargo de personas concretas, no como una actividad externalizada o coreografiada. Consiste en la defensa de los intereses mediante el empleo de herramientas propias del ejercicio diplomático, por lo que tienen que incluirse en el catálogo de actividades internas y en la cúspide de la tarea directiva. Por ser más precisos, la diplomacia corporativa es una función atribuida a un grupo concreto de personas, que tienen sobre sí la responsabilidad de coordinar las herramientas y expandir la cultura diplomática en la organización.

Esta configuración de la diplomacia corporativa se alinea con el enfoque Morgesson y Hofmann (1999), quienes consideran que las empresas se han convertido en instituciones antropomórficas. Los atributos de los directivos y los empleados se vinculan a los productos y los servicios ofertados por las compañías y, en consecuencia, a los resultados económicos de las mismas. Para den Hond et al. (2014) tanto en el caso de la actividad política de la compañía como en el desarrollo e implementación de la responsabilidad social se puede inferir un nuevo tipo de problema propio de la teoría de la agencia. Los comportamientos y los intereses de los directivos afectan de forma negativa en la reputación de la compañía. Por este motivo, Saner, Yiu y Sondergaard (2000) consideran que el éxito de las compañías globales está estrechamente asociado a la capacidad de sus directivos de desarrollar nuevas competencias.

\section{La tarea de la diplomacia comporativa consiste en la defensa de los intereses mediante el empleo de herramientas propias del ejercicio diplomático}




\section{Cuatro funciones de la actividad diplomática corporativa}

La diplomacia corporativa adquiere rango sustantivo cuando se asimila a las cuatro funciones básicas de la actividad diplomática: la representación del Estado, la protección de los intereses tanto del Estado acreditante como de los nacionales dentro del derecho, la negociación con el Gobierno, la recogida de información sobre los acontecimientos locales por medios lícitos y su transmisión, y el fomento de las relaciones amistosas. Junto al derecho internacional basado en la costumbre, la Convención de Viena sobre Relaciones Diplomáticas (1961) señala esas funciones de la misión diplomática, cuyo núcleo se puede trasladar a la actividad privada.

La representación es la primera de las funciones. Hasta ahora, los máximos dirigentes empresariales, ya fuesen como ejecutivos o como representantes del capital de la empresa han tenido mayoritariamente un papel orientado casi exclusivamente a la gestión interna, con pequeñas incursiones en el ámbito institucional y la opinión pública. Este papel tradicional resulta insuficiente en el panorama actual, donde el papel del máximo representante corporativo juega un papel de liderazgo mucho más político y visible. Ya no es posible empujar el desarrollo de los objetivos empresariales concediendo alguna que otra entrevista o mediante un discurso anual ante la junta general de accionistas. La cuenta de resultados depende ahora de más elementos y la reputación que se decide en el marco de la comunicación pública es, desde luego, uno de ellos. En el zoco de las ideas, nuestra propuesta tiene que ser diferenciadora con argumentos y hechos veraces, no solo con acciones aisladas.

La representación no puede delegarse en la embajada o en la oficina comercial porque la titularidad de los negocios internacionales suele ser dispersa. Más aún, Kesteleyn, Riordan y Ruël (2014) recuerdan "la nacionalidad de las compañías ha devenido más fluida, de modo que se torna complejo determinar a qué gobierno o a qué embajada le corresponda prestar ayuda” (p. 305). En cambio, "una regla es inmutable: los intereses de un país son de carácter permanente. Las compañías que buscan el apoyo de sus países originarios para afrontar un desafío en la gestión de sus negocios olvidan este principio bajo su propio riesgo” (Small, 2014, p. 378). Jade Miller (2009) enfatiza el enfoque de la economía política con un discurso propio: "el Estado debe estar atento a cómo la diplomacia convencional entre las corporaciones de sus países y los gobiernos extranjeros afectan a su propia imagen y reputación" (p. 286) y señala que "las corporaciones multinacionales de Estados Unidos han estado implicadas en numerosas prácticas que podrían considerarse dudosas en relación con la política exterior estadounidense” (p. 288).

La negociación consiste en la capacidad de llegar a acuerdos sostenibles en el tiempo y que sean capaces de generar ventajas competitivas concretas, sólidas. La función diplomática pivota sobre la capacidad de interlocución con distintos grupos de interés que, probablemente, presentan problemas y voluntades divergentes. Aun en caso de conflicto evidente, la diplomacia corporativa contribuye a la creación de redes de interés propio y destrucción de las ajenas. Small (2014) apunta que "el gobierno se apunta a la negociación cuando comprende el beneficio político de sentarse a la mesa para negociar una solución antes que continuar siendo el principal obstáculo de un acuerdo” (p. 390).

Aquí es fundamental la dimensión humana, la capacidad de entender los asuntos en clave personal. El rol que desempeña el directivo responsable de la negociación es fundamental. La personalidad del primer directivo es un activo diplomático que varía según la naturaleza de la compañía. Este elemento antropomórfico antes mencionado determina cómo se establecen las
El panorama actual, el papel del máximo representante corporativojuega un papel de liderazgo mucho más político y visible 
relaciones y cómo se gestionan los recursos en cada momento. La gestión de la influencia ha perdido su carácter privado o bidireccional. El entorno complejo reclama la participación de terceros en beneficio propio mediante el tejido de alianzas o redes de trabajo. En un país extranjero, el apoyo de terceros es esencial para el éxito de las campañas. Small (2014) denomina esta táctica "triangular los intereses" y sostiene que "mediante la inclusión del apoyo de terceros, la compañía y el gobierno de tu país [de origen] hacen un esfuerzo para alterar el balance asimétrico del poder del gobierno local" (pp. 387-388). Esta triangulación adquiere peso específico en las organizaciones multilaterales.

La diplomacia corporativa no es una actividad sinónimo de lobby y, aquí, discrepo con las ideas de Susan Strange (2000). La académica británica pone el acento en la relación entre las empresas y los gobiernos, una interpretación que deja fuera algunos elementos del estado actual, la estrategia y la gestión internacional de los negocios. En su relación con los gobiernos, las organizaciones tienen como objetivo influir en las políticas económicas e industriales de un país. Una segunda modalidad puede ser el establecimiento de relaciones con otras empresas del sector con la finalidad de establecer alianzas, generar coaliciones o emprender nuevos proyectos. Strange relaciona esta actividad con el lobby en la medida que señala la presión sobre el legislador como una medida recurrente. Así, se puede influir en la regulación en uno u otro sentido. Este enfoque del cabildeo excluye a otras numerosas organizaciones que persiguen los mismos fines y emplean esos medios.

Strange acierta cuando apunta la necesidad de saber gestionar las relaciones con los reguladores en sus distintos niveles de actuación (local, nacional o internacional). En concreto, consiste en conocer cuáles son las necesidades del legislador y del entorno afectado, así como la interpretación de las tendencias de cambio. En otro orden, es necesario conocer cómo influyen los sindicatos globales en las decisiones laborales. Según la industria o el mercado, su capacidad de influencia en las negociaciones puede ser relevante. Por eso, no es función exclusiva de las grandes corporaciones, sino que está presente también en pequeñas y medianas empresas (Romero \& Rodríguez-Gutiérrez, 2014).

Ese conocimiento del medio en el que se desenvuelve una compañía puede ser la única vía para anticiparse a los cambios. Por eso, en mi opinión, no es solo el seguimiento de la actividad legislativa, sino también una actuación preventiva, que contribuya a reducir los riesgos o los conflictos con la administración pública. La actividad de seguimiento es reactiva y cede el liderazgo a terceros, con el consecuente incremento de la incertidumbre. Resulta más competitivo el conocimiento exacto de la evolución política y social de las instancias claves que una organización debe tener en cuenta cuando diseña su estrategia. En esto consiste la inteligencia directiva.

La protección se concreta en dos áreas de la organización. En primer lugar, las personas, que son el motor de la internacionalización. La experiencia diplomática tiene que servir para tejer una red de profesionales que combine las decisiones estratégicas del centro con su aplicación táctica en cada uno de los mercados destino. Cada país reclama una fórmula diferenciada. Más aún, la protección tiene que ver con la salida del país en caso de emergencia, la capacidad de conectar con los agentes de policía y defensa del país, el conocimiento del funcionamiento de los servicios de inteligencia. Kesteleyn, Riordan y Ruël (2014) asemejan esta labor a la propia de los servicios consulares: "evacuación del equipo humano en situaciones de

\section{La diplomacia comorativa no es una actividad sinónimo de lobby}


crisis y, más aún, en actividades que se asemejan a las operaciones militares” (p. 306) como la protección contra la piratería.

El segundo eje de protección es la cadena de suministros globales (Romero, 2009). No importa la actividad: los procesos mundiales de producción, distribución y consumo conectan los bloques geográficos de libre comercio. Es necesario proteger los distintos estadios para no verse sobrepasado por una crisis, una emergencia, un corte de suministro o la subida del nivel del mar. Esta visión global de los recursos amplía los requerimientos de los sistemas de seguridad y orienta la inteligencia directiva hacia la protección de aquello que es insustituible: las personas y las materias primas. Small (2014) incide en la complejidad de las relaciones entre empresa internacional y gobierno local: el primer paso consiste en "reconciliar el deseo de una compañía de obtener ayuda en un tercer país con los intereses nacionales permanentes de este” (p. 381).

La promoción de los intereses, la misión, los productos y los servicios de la compañía tiene distintas dimensiones. En el ámbito del marketing, se concreta en el conocimiento de los gustos y los patrones de consumo, el análisis de las tendencias, el juicio de la opinión pública, la comprensión de las dinámicas comerciales. En el plano de la comunicación estratégica, la transparencia, la comunicación instantánea, las redes sociales y los nuevos medios han convertido a las empresas y a sus dirigentes en personajes públicos con capacidad de influir en la percepción que se tiene de las compañías que dirigen. Por eso, la diplomacia corporativa incide en la gestión de la información pública, la relación con los medios de comunicación, la capacidad de hablar en público con elocuencia, la habilidad de producir y distribuir mensajes periodísticos, la relación con los blogueros y las redes sociales, entre otras tareas. La promoción, unida a la negociación, se manifiesta en la empatía, la comunicación intercultural, el protocolo, entre otras ocupaciones. Los think tanks emergen como espacio de intermediación con los poderes públicos y son cada vez más relevantes en la conformación de los asuntos públicos (Manfredi et al., 2015).

\section{La diplomacia corporativa y la innovación directiva}

La diplomacia corporativa es una función propia de la innovación directiva en el siglo XXI. La presencia en la arena pública, así como la afectación de la cuenta de resultados por las acciones que en ella se ejecutan, han dado un nuevo sentido a la idea de colaboración y relación con terceros. Es un salto cualitativo en la teoría de los stakeholders, tan en boga en los años noventa. Tras la publicación del libro de R. E. Freeman Strategic Management: A Stakeholder Approach en 1984, numerosas empresas y analistas comprendieron las externalidades, la gestión de las relaciones con terceros y la necesidad de diseñar una estrategia que tenga en cuenta qué consecuencias tienen nuestras decisiones empresariales. La transformación concede más valor a la opinión pública, tan presente en cualquier decisión. Cualquier afectado, ataviado con su teléfono móvil y su cámara portátil, puede dar cuenta de un comportamiento no adecuado. El ámbito público de la compañía es más extenso y, desde luego, no está bajo el control exclusivo de la firma.

Negociar con los agentes externos, establecer alianzas estratégicas en distintas partes del mundo, maniobrar mediante coaliciones ofensivas o defensivas, tener la mejor información disponible sobre aquellos aspectos considerados claves para la toma de decisiones es todo un reto cuando la delimitación del mercado ya no se circunscribe al mercado doméstico y a unos cuantos países fuera de las fronteras nacionales. La nueva sociedad globalizada multiplica exponencialmente la necesidad de un caudal de información constante, de una presencia continuada y 
de unas herramientas de gestión de probada eficacia. Asimismo, la transparencia empresarial se ha convertido en un aspecto esencial para operar en los mercados. Ha crecido la demanda de responsabilidad empresarial bajo distintas fórmulas.

El perfil del líder empresarial con poder absoluto ha devenido en la necesidad de un nuevo liderazgo mucho más centrado en el entendimiento, la capacidad de persuasión y la eficacia en la búsqueda de consensos asumibles, en definitiva, todo el elenco de características que componen la inteligencia directiva. Igual que el poder duro ha evolucionado hacia nuevos territorios, la autoridad del primer directivo ha mutado hacia otros ámbitos. Se requiere empatía, capacidad de escucha y comprensión de la diversidad en la globalización. La diplomacia corporativa, por tanto, nace para cubrir las necesidades de liderazgo en las organizaciones globales, como ha sucedido en la gestión de los países (La Porte, 2007). Las corporaciones han tomado conciencia de su mayor autonomía frente a los poderes públicos y de las carencias de estos últimos para contribuir rápida y diligentemente en la resolución de sus conflictos y crisis en otros mercados siguiendo el patrón propio de la diplomacia internacional y, al tiempo, de los servicios diplomáticos nacionales. De entrada, todos aquellos que contribuyen a la reputación, a la percepción y a la credibilidad de las empresas propiciando un conocimiento acorde con la realidad y un diálogo fluido con todos los agentes sociales implicados.

Es un contexto muy complejo. El diplomático corporativo actúa como un director de orquesta que busca la armonía. Se guía por la inteligencia directiva, no solo por la cuenta de resultados o la burbuja del resultado de cada trimestre. Este tipo de liderazgo favorece el presidencialismo, el protagonismo del empresario que proyecta su personalidad, teje una red de contactos al servicio del negocio y que genera un entorno de confianza. La alta dirección acompaña las decisiones, pero si se pretende que la diplomacia privada sea exitosa debe contar con el respaldo y la ejecución del primer ejecutivo.

En suma, el directivo, en cuanto a diplomacia corporativa se refiere, se convierte en un estadista, una persona capaz de aunar voluntades. En un mercado abierto y competitivo, el empresario/punta de lanza juega a otro juego, aquel que trata de estar más informado, mejor posicionado y mejor reputado. La diplomacia corporativa puede contribuir a la innovación en el modelo de gestión y dotar a los consejos de administración de herramientas para dar el salto la economía global.

\section{Referencias}

Baron, D. R. (2003). Business and its environment. New York: Prentice-Hall.

Bayne, N., \& Woolcock, S. (2007). The New Economic Diplomacy: Decision-making and negotiation in international economic relations. Aldershot: Ashgate.

Berridge, G. R. (2015). Diplomacy. Theory and Practice. London: Palgrave MacMillan.

Den Hond, F., Rehbein, K. A., Bakker, F. G. A. de, \& van Lankveld, H. (2014). Playing on two chessboards: Reputation effects between Corporate Social Responsibility (CSR) and Corporate Political Activity (CPA). Journal of Management Studies, 51(5), 790-813.

Deutsch, K. (1978). The Analysis of International Relations. Englewood Cliffs, NJ: Prentice-Hall.

Freeman, E. (1984). Strategic Management. A Stakeholder Approach. Boston: Pittman.

Huang, C. J. (2010). Corporate governance, corporate social responsibility and corporate performance. Journal of Management E Organization, 16(5), 641-655.
El directivo, en

cuanto a

diplomacia

comporativa se

refiere, se convierte

en un estadista,

una persona capaz

de aunar

voluntades 
Kesteleyn, J., Riordan, S., \& Ruël, H. (2014). Introduction: Business Diplomacy. The Hague journal of diplomacy, 9(4), 303-309.

L’Etang, J. (2008). Public Relations: Concepts, Practice, and Critique. London: Sage.

La Porte, T. (2007). Diplomacia pública americana: lecciones para una comunicación política internacional. Communication and Society/Comunicación y Sociedad, XX(2), 23-59.

Lee, D. (2004). The growing influence of business in UK diplomacy. International Studies Perspectives, 5, 50-54.

Manfredi Sánchez, J. L. (2011). Hacia una teoría comunicativa de la diplomacia pública. Communication and Society/Comunicación y Sociedad, XXIV(2), 150-166.

Manfredi Sánchez, J. L. (2014). Taxonomía de la diplomacia digital en la agenda de las nuevas relaciones internacionales. Historia y Comunicación Social, 19, 341-354.

Manfredi Sánchez, J. L., Sánchez-Giménez, J. A., \& Pizarro-Miranda, J. (2015). Structural Analysis to Measure the Influence of Think Tanks' Networks in the Digital Era. The Hague Journal of Diplomacy, 10(4), 363-395.

Miller, J. (2009). Soft-Power and State-Firm Diplomacy: Congress and IT Corporate Activity in China. International Studies Perspectives, 10, 285-302.

Morgeson, F. P., \& Hofmann, D. A. (1999). The structure and function of collective constructs: Implications for multilevel research and theory development. Academy of Management Review, 24, 249-265.

Ordeix-Rigo, E., \& Duarte, J. (2009). From Public Diplomacy to Corporate Diplomacy: Increasing Corporation's Legitimacy and Influence. American Behavioral Scientist, 53, 549-564.

Romero, I. (2009). Pymes y Cadenas de Valor Globales. Implicaciones para la política industrial en las economías en desarrollo. Análisis Económico, XXIV(57), 199-216.

Romero, I., \& Rodríguez-Gutiérrez, M. J. (2014). Sobre la internacionalización de la pyme y su contribución a la imagen exterior de España. Comillas Journal of International Relations, 1, 91-104.

Ruël, H. J. M. (2013). Diplomacy means Business. Zwolle: Windesheim.

Ruël, H. J. M., \& Wolters, T. (2016). Business Diplomacy. En C. M. Constantinou, P. Kerr \& P. Sharp (Eds.), The Sage Handbook of Diplomacy (capítulo 46). London: SAGE Publications Ltd.

Saner, R., \& Yiu, L. (2005). Swiss Executives as Business Diplomats in the New Europe: Evidence from Swiss Pharmaceutical and Agro-Industrial Global Companies. Organizational Dynamics, 34(3), 298-312.

Saner, R., Yiu, L., \& Søndergaard, M. (2000). Business diplomacy management: A core competency for global companies, The Academy of Management Executive, 14(1), 80-92.

Small, J. M. (2014). Business Diplomacy in practice: Advancing Interests in Crisis Situations. The Hague Journal of Diplomacy, 9, 374-392.

Søndergaard, M. (2014). Corporate Business Diplomacy: Reflections on the Interdisciplinary Nature of the Field. The Hague Journal of Diplomacy, 9, 356-371.

Strange, S. (1998). State and Markets. London: Pinter Publishers. 